\section{Factors Affecting Seed Germination of Ilex latifolia and I. rotunda}

\author{
Takahiro Tezuka, Hisa Yokoyama, Hideyuki Tanaka, Shuji Shiozaki, \\ and Masayuki Oda ${ }^{1}$ \\ Graduate School of Life and Environmental Sciences, Osaka Prefecture \\ University, Sakai, Osaka 599-8531, Japan
}

Additional index words. cold stratification, cut seeds, dormancy, embryo culture, water permeability

\begin{abstract}
Mature seeds of Ilex species usually contain immature embryos and are extremely difficult to germinate. Ilex latifolia and I. rotunda, two species that are grown as ornamentals, also produce seeds that are difficult to germinate. In the present study, we investigated some factors affecting seed germination in those species. Although seeds of $I$. latifolia and $I$. rotunda could imbibe water, they did not germinate. When embryos were cultured in vitro, germination was observed in I. latifolia but not in I. rotunda. Interestingly, a transient decrease in germination frequency occurred in I. latifolia embryos isolated from seeds collected in September or October. Among five types of I. latifolia seeds that differed in the presence of the endocarp, testa, and endosperm, germinability of isolated embryos was highest. Good germination was also observed in quarter-seeds with or without endocarp followed by half-seeds. Treatment of seeds with sodium hydroxide $(\mathrm{NaOH})$ had no effect on seed germination in I. latifolia. Cold stratification at $5{ }^{\circ} \mathrm{C}$ increased the germination frequency of I. latifolia embryos. In conclusion, the data suggested a mechanical barrier by the endocarp and inhibitors contained in the endosperm, testa, and/or endocarp inhibited seed germination in I. latifolia. Although no seeds or embryos of $I$. rotunda germinated, cold stratification in combination with other treatments deserves further investigation. Chemical name: sodium hydroxide $(\mathrm{NaOH})$.
\end{abstract}

The genus Ilex (Aquifoliaceae) consists of more than 500 species of dioecious trees and shrubs distributed throughout the temperate and tropical regions of the world (Galle, 1997). Some species are grown as ornamentals. In Japan, Ilex latifolia is planted in temples and shrines, and I. rotunda is grown as garden, park, and street trees. Ilex latifolia and I. rotunda (Goi et al., 1978) flower in May and June, and their fruits mature to red in the fall (around November). Leaves of I. latifolia contain high levels of flavonoids and have been used for beverage tea in China (Liang et al., 2001).

Although vegetative propagation methods such as tissue culture are sometimes used to propagate Ilex species (Luna et al., 2003; Mattis et al., 1995; Rey et al., 2002), the simplicity of reproduction through seeds has advantages for mass propagation. However, Ilex seeds are difficult to germinate, because embryos in mature seeds are usually at the immature heart or torpedo stage (Chien et al., 2011; Hu et al., 1979; Tsang and Corlett, 2005). This immaturity is thought to be caused by dormancy rather than extremely slow growth (Hu et al., 1979).

Embryo culture techniques have been used to overcome seed dormancy (Biggs et al., 1986; Chee, 1994; Raghavan, 2003; Sharma

Received for publication 16 Nov. 2012. Accepted for publication 24 Jan. 2013.

${ }^{1}$ To whom reprint requests should be addressed; e-mailmoda@plant.osakafu-u.ac.jp. et al., 1996) and to shorten germination time (Acebedo et al., 1997; Tezuka et al., 2012) in many plant species. Seedlings have also been obtained for several Ilex species by culturing immature embryos (Hu, 1975; Mattis et al., 1995; Sansberro et al., 1998, 2001). Hu et al. (1979) reported that when isolated embryos of I. aquifolium, I. cornuta, and I. opaca were cultured in vitro, most of them developed at the mature stage and germinated, whereas embryos with endosperm showed only slight embryonic growth. Based on these results, they suggested that inhibitors responsible for maintaining embryo dormancy in the seed exist in the endosperm and/or membrane-like testa attached to the endosperm. Conversely, in I. paraguariensis, no difference was observed in germination frequency between embryos and bisected pyrenes (seeds), suggesting that the endosperm did not inhibit embryo development or germination (Dolce et al., 2010). In I. dumosa, germination frequency of cut pyrenes was higher than that of embryos (Dolce et al., 2011). Therefore, different mechanisms may be involved in seed dormancy among Ilex species.

The main objective of the present study was to identify factors affecting seed germination in I. latifolia and I. rotunda. We investigated the germination capacity of seeds and embryos of those species from fruits collected in different months. We also investigated the effect of the endosperm, testa, and endocarp on germination using cut seeds. In addition, we tested seed water permeability and the effect of several treatments on seed germination, including $\mathrm{NaOH}$ (Crisosto and Sutter, 1985; Sun et al., 2006), gibberellic acid $\left(\mathrm{GA}_{3}\right.$; Chen et al., 2008, 2010; Nicolás et al., 1996), and a moist, cold storage (cold stratification; Abe and Matsunaga, 2011; Chien et al., 1998; Sun et al., 2006; Tsuyuzaki and Miyoshi, 2009), all of which have been used to break seed dormancy in many plant species.

\section{Materials and Methods}

Plant materials. Fruits were harvested from I. latifolia and I. rotunda plants grown in a field at Osaka Prefecture University, Osaka, Japan. Seeds were washed with water to remove the fruit pulp before use in the experiments.

Germination test of seeds and embryos. Fruits of I. latifolia and I. rotunda were collected on 6 Feb. 2007. Non-sterilized seeds were sown in petri dishes that contained wet filter papers and were incubated at $15{ }^{\circ} \mathrm{C}$ in the dark. After two months (9 Apr.), the seeds were then transferred to a 288-cell tray $(10 \mathrm{~mL} /$ cell $)$ filled with wet vermiculite and placed in a greenhouse. Seeds were observed for emergence for one year after sowing.

To culture embryos, I. latifolia seeds collected on 9 Jan. 2007 and I. rotunda seeds collected on 12 Jan. 2007 were sterilized with $70 \%$ ethanol for $30 \mathrm{~s}$ and then with 5\% sodium hypochlorite for $15 \mathrm{~min}$. Embryos were excised from the sterilized seeds, placed in flat-bottomed test tubes $(25 \mathrm{~mm}$ diameter, $100 \mathrm{~mm}$ length) that contained $8 \mathrm{~mL}$ of plant growth regulator-free Murashige and Skoog (MS) medium (Murashige and Skoog, 1962) supplemented with $3 \%$ sucrose and $0.85 \%$ agar $(\mathrm{pH} \mathrm{5.8)}$ and that were autoclaved for $15 \mathrm{~min}$ at $121^{\circ} \mathrm{C}$, and then incubated at $25^{\circ} \mathrm{C}$ in the dark. Embryos were observed for germination for six months.

Effect of seed developmental stage on germination capacity. Fruits collected at monthly intervals in two seasons (July 2006 to Feb. 2007 and July to Dec. 2007; see Table 2) were used to investigate whether the germination capacities of seeds and embryos were correlated with the seed developmental stage. In the 2006-07 season, non-sterilized seeds were sown in petri dishes containing wet filter paper and were incubated at $25^{\circ} \mathrm{C}$ in the dark. Other seeds (except I. latifolia seeds collected on 18 July) were used for embryo culture at $25^{\circ} \mathrm{C}$ in the dark as described previously. We could not isolate embryos from I. latifolia seeds collected on 18 July because the embryos were immature and too small to excise. Therefore, those seeds were cut in half, and the half-seeds with embryos (Fig. 1B) were aseptically sown. In the 2007 season, non-sterilized seeds were sown as described previously and incubated at $15^{\circ} \mathrm{C}$ in the dark. Embryos were excised from the sterilized seeds (excluding I. latifolia seeds collected on $20 \mathrm{July}$ ), placed in flat-bottomed test tubes that contained MS medium supplemented with the cytokinin 6-benzyladenine (BA; 0 or $\left.1 \mathrm{mg} \cdot \mathrm{L}^{-1}\right)$, the auxin 1-naphthaleneacetic 
Table 1. Germination of seeds and embryos of Ilex latifolia and I. rotunda.

\begin{tabular}{|c|c|c|c|}
\hline Species & Method & $\begin{array}{c}\text { Emergence or } \\
\text { germination }(\%)\end{array}$ & $\begin{array}{c}\text { Number of days to } \\
\text { emergence or germination }\end{array}$ \\
\hline \multirow[t]{2}{*}{ I. latifolia } & Seed sowing ${ }^{z}$ & 0 & - \\
\hline & Embryo culture ${ }^{y}$ & 100 & $18.9 \pm 1.1$ \\
\hline \multirow[t]{2}{*}{ I. rotunda } & Seed sowing ${ }^{z}$ & 0 & 一 \\
\hline & Embryo culture & 0 & - \\
\hline
\end{tabular}

${ }^{\mathrm{z}} \mathrm{n}=30$. Observations were continued for one year after sowing.

${ }^{\mathrm{y}} \mathrm{n}=10$. Observations were continued for six months after incubation.

Data are expressed as mean $\pm \mathrm{SE}$.

Table 2. Effect of seed developmental stage on germination capacity of seeds and embryos of Ilex latifolia and $I$. rotunda.

\begin{tabular}{|c|c|c|c|c|c|c|}
\hline \multirow[b]{4}{*}{ Species } & & & \multirow{4}{*}{$\begin{array}{l}\text { Length of embryo } \\
(\mu \mathrm{m})^{\mathrm{z}}\end{array}$} & \multicolumn{3}{|c|}{ Germination $(\%)^{\mathrm{y}}$} \\
\hline & & & & \multirow[b]{3}{*}{ Seed } & \multicolumn{2}{|c|}{ Embryo } \\
\hline & \multicolumn{2}{|c|}{ Date of seed collection } & & & & $1 \mathrm{mg} \cdot \mathrm{L}^{-1} \mathrm{BA}$ and \\
\hline & Season & Date & & & PGR-free & $0.1 \mathrm{mg} \cdot \mathrm{L}^{-1} \mathrm{NAA}$ \\
\hline \multirow[t]{14}{*}{ I. latifolia } & $2006-07$ & 18 July & - & 0 & $0^{\mathrm{x}}$ & - \\
\hline & & 18 Aug. & $373 \pm 15 a$ & 0 & 70 & - \\
\hline & & 18 Sept. & $379 \pm 19 a$ & 0 & 0 & - \\
\hline & & 12 Oct. & $483 \pm 18 b c$ & 0 & 100 & - \\
\hline & & 16 Nov. & $484 \pm 22 b c$ & 0 & 90 & - \\
\hline & & 12 Dec. & $471 \pm 11 b$ & 0 & 100 & - \\
\hline & & 12 Jan. & $544 \pm 20 \mathrm{bc}$ & 0 & 100 & - \\
\hline & & 12 Feb. & $551 \pm 13 \mathrm{c}$ & 0 & 90 & - \\
\hline & 2007 & 20 July & - & 0 & $40^{\mathrm{w}}$ & $0^{\mathrm{w}}$ \\
\hline & & 7 Aug. & $309 \pm 13 a$ & 0 & 10 & 0 \\
\hline & & 5 Sept. & $419 \pm 21 b$ & 0 & 60 & 40 \\
\hline & & 4 Oct. & $488 \pm 11 \mathrm{c}$ & 0 & 20 & 20 \\
\hline & & 2 Nov. & $465 \pm 12 b c$ & 0 & 100 & 60 \\
\hline & & 11 Dec. & $456 \pm 15 b c$ & 0 & 80 & - \\
\hline \multirow[t]{10}{*}{ I. rotunda } & 2006-07 & 6 Sept. & $194 \pm 6 \mathrm{a}$ & 0 & 0 & - \\
\hline & & 6 Oct. & $261 \pm 10 b$ & 0 & 0 & - \\
\hline & & 6 Nov. & $292 \pm 6$ bc & 0 & 0 & - \\
\hline & & 6 Dec. & $295 \pm 10 b c$ & 0 & 0 & - \\
\hline & & 7 Jan. & $318 \pm 11 \mathrm{c}$ & 0 & 0 & - \\
\hline & & 7 Feb. & $289 \pm 14 b c$ & 0 & 0 & - \\
\hline & 2007 & 5 Sept. & $282 \pm 6 \mathrm{a}$ & 0 & 0 & 0 \\
\hline & & 4 Oct. & $300 \pm 8 a b$ & 0 & 0 & 0 \\
\hline & & 2 Nov. & $302 \pm 5 a b$ & 0 & 0 & 0 \\
\hline & & 11 Dec. & $313 \pm 6 b$ & 0 & 0 & 0 \\
\hline
\end{tabular}

${ }^{\mathrm{z}}$ Data are expressed as mean \pm SE $(\mathrm{n}=10)$. Means followed by different letters are significantly different $(P<0.05$, one-way analysis of variance with Tukey's post hoc test) within a season in each species.

${ }^{y}$ Seeds and embryos in the 2006-07 and 2007 seasons $(n=10$ in each treatment) were incubated at 25 and $15^{\circ} \mathrm{C}$, respectively. Observations of seeds and embryos were continued for one year after sowing and 20 weeks after incubation, respectively.

'Ilex latifolia seeds harvested on 18 July 2006 were cut in half, and halves containing embryos were sown in vitro, because the embryos were too small to isolate.

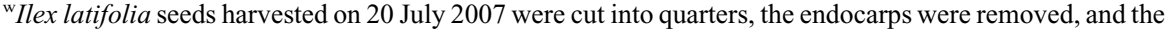
quarters containing embryos were sown in vitro, because the embryos were too small to isolate.

A



B

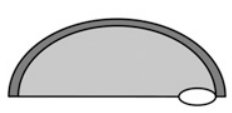

C

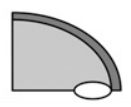

D

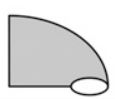

Fig. 1. Schematic illustration of five types of Ilex latifolia seeds used to investigate the effects of endosperm, testa, and endocarp on seed germination. (A) Intact seed; (B) half-seed; (C) quarter-seed; (D) quarter-seed without endocarp; (E) isolated embryo (seed without endocarp, testa, or endosperm).

acid (NAA; 0 or $0.1 \mathrm{mg} \cdot \mathrm{L}^{-1}$ ), 3\% sucrose, and $0.85 \%$ agar $(\mathrm{pH} \mathrm{5.8)}$, and then incubated at $15^{\circ} \mathrm{C}$ in the dark. Ilex latifolia seeds harvested on 20 July were cut into quarters, and the endocarps were removed. The quarterseeds with embryos but not endocarps (Fig. 1D) were aseptically sown. Embryo length was measured using a stereomicroscope (SZ60;
Olympus, Tokyo, Japan) equipped with an ocular micrometer, when embryos were cultured. Germination of seeds and embryos were observed for one year after sowing and 20 weeks after incubation, respectively.

Water permeability test. Methylene blue has been used to determine the water permeability of seeds (Orozco-Segovia et al., 2007). Seeds of I. latifolia (collected 14 Nov. 2007) and $I$. rotunda (collected 16 Nov. 2007) were immersed for $48 \mathrm{~h}$ in $1 \%$ methylene blue or distilled water (control). The seeds were washed with water, cut, and observed with a stereomicroscope (SZ60; Olympus).

Effects of endosperm and endocarp on seed germination. Ilex latifolia seeds (collected 8 Nov. 2007) were sterilized with $70 \%$ ethanol for $30 \mathrm{~s}$ and then with 5\% sodium hypochlorite for $15 \mathrm{~min}$. Five types of explants were prepared: intact seeds (Fig. 1A), half-seeds (Fig. 1B), quarter-seeds (Fig. 1C), quarter-seeds without endocarp (Fig. 1D), and isolated embryos (seeds without endocarp, testa, or endosperm; Fig. 1E). These explants were placed in flat-bottomed test tubes containing $8 \mathrm{~mL}$ of MS medium supplemented with $3 \%$ sucrose and $0.85 \%$ agar (pH 5.8) and then incubated at $15{ }^{\circ} \mathrm{C}$ in the dark. Seed germination was observed for 10 weeks after incubation.

Effect of chemical scarification on seed germination. Ilex latifolia seeds (collected 12 Oct. 2006) were immersed in $1 \mathrm{M} \mathrm{NaOH}$ for $3 \mathrm{~h}$. These seeds were washed with water, sown in petri dishes that contained wet filter papers, and incubated at $25{ }^{\circ} \mathrm{C}$ in the dark. Observations of seed germination were recorded for six months after sowing.

Effect of gibberellic acid on seed germination. Ilex latifolia and $I$. rotunda seeds (collected 6 Feb. 2007) were immersed in $100 \mathrm{mg} \cdot \mathrm{L}^{-1} \mathrm{GA}_{3}$ for $24 \mathrm{~h}$ and then placed in petri dishes containing wet filter papers. These seeds were incubated at $15^{\circ} \mathrm{C}$ in the dark and observed for six months. After incubation, embryo length was measured using a stereomicroscope (SZ60; Olympus) equipped with an ocular micrometer.

Effect of cold stratification on seed germination. Ilex latifolia and $I$. rotunda seeds (collected 6 Feb. 2007) were placed in petri dishes that contained wet filter papers, incubated at $15^{\circ} \mathrm{C}$ in the dark for 14 weeks (until $15 \mathrm{May}$ ), and then transferred to $5^{\circ} \mathrm{C}$ in the dark. Finally, the seeds were transferred to an incubator maintained at $15{ }^{\circ} \mathrm{C}$ in the dark at $\approx 6$-week intervals (22 June, 7 Aug., 18 Sept., 30 Oct., and 4 Dec.; see Table 3). After 44 weeks of treatment, some seeds were continuously incubated at $15^{\circ} \mathrm{C}$ in the dark and other seeds were used for embryo culture at $15^{\circ} \mathrm{C}$ in the dark. Embryo length was measured using a stereomicroscope (SZ60; Olympus) equipped with an ocular micrometer, when embryos were cultured. Germination of seeds and embryos was observed for an additional 10 and 8 weeks, respectively.

Statistical analysis. For the analysis of embryo length, one-way analysis of variance with Tukey's honestly significant difference 
post hoc test (SPSS Statistics 20; IBM Japan,

\section{Results}

Germination test of seeds and embryos. When non-sterilized seeds of I. latifolia and I. rotunda were sown on wet filter paper at $15{ }^{\circ} \mathrm{C}$ and then transferred to a greenhouse, they did not germinate even one year after sowing. When embryos were cultured in vitro, all I. latifolia embryos germinated by $18.9 \pm$ $1.1 \mathrm{~d}$ after incubation at $25^{\circ} \mathrm{C}$. Meanwhile, I. rotunda embryos did not germinate (Table 1).

Effect of seed developmental stage on germination capacity. Embryos of I. latifolia in the 2006-07 season were $373 \pm 15 \mu \mathrm{m}$ long on 18 Aug. 2006 and increased to $483 \pm 18 \mu \mathrm{m}$ by 12 Oct. 2006. In 2007 season, embryos also elongated through October, suggesting that I. latifolia embryos developed at least until October. The length of I. rotunda embryos increased approximately until November in either the 2006-07 or 2007 seasons (Table 2).

When non-sterilized seeds of I. latifolia and I. rotunda were sown on wet filter paper, germination was not observed in either the Tokyo, Japan) was performed.

2006-07 or 2007 seasons. In contrast, when cut seeds or embryos were cultured in vitro, quarter-seeds without endocarp (sown on 20 July 2007) and embryos of I. latifolia germinated, whereas germination was not observed in I. rotunda. Interestingly, although the germination frequency of $I$. latifolia embryos collected on 18 Aug. 2006 was 70\%, it decreased to $0 \%$ in those collected on 18 Sept. and increased to $100 \%$ in those collected on 12 Oct. Similar transient decreases in germination was also observed on 4 Oct. 2007. Adding BA and NAA to MS medium did not improve germination frequency (Table 2).

Water permeability test. Intact seeds of I. latifolia and I. rotunda were immersed in methylene blue to investigate seed water permeability. In both species, although the embryo, endosperm, and most of the testa were not stained, the endocarp and testa adjacent to the embryo were stained (Fig. 2). These results suggested that seeds of $I$. latifolia and I. rotunda could imbibe water.

Effects of endosperm and endocarp on seed germination. To test the hypothesis that inhibitors present in the endosperm, testa, and/or endocarp interfere with seed

Table 3. Effect of cold stratification on germination of seeds and embryos of Ilex latifolia and I. rotunda.

\begin{tabular}{|c|c|c|c|c|c|c|}
\hline \multirow[b]{2}{*}{ Species } & \multicolumn{3}{|c|}{ Treatment period (weeks) } & \multirow[b]{2}{*}{ Length of embryo $(\mu \mathrm{m})^{\mathrm{x}}$} & \multicolumn{2}{|c|}{ Germination (\%) } \\
\hline & $15^{\circ} \mathrm{C}$ & $5^{\circ} \mathrm{C}$ & $15^{\circ} \mathrm{C}$ & & Seed & Embryo \\
\hline \multirow[t]{6}{*}{ I. latifolia ${ }^{2}$} & 14 & 0 & 30 & $529 \pm 15 \mathrm{a}$ & 0 & 50 \\
\hline & 14 & 6 & 24 & $506 \pm 19 a$ & 0 & 50 \\
\hline & 14 & 12 & 18 & $549 \pm 16 \mathrm{a}$ & 0 & 60 \\
\hline & 14 & 18 & 12 & $517 \pm 24 \mathrm{a}$ & 0 & 70 \\
\hline & 14 & 24 & 6 & $522 \pm 15 \mathrm{a}$ & 0 & 80 \\
\hline & 14 & 30 & 0 & $532 \pm 21 \mathrm{a}$ & 0 & 70 \\
\hline \multirow{6}{*}{ I. rotund $a^{\mathrm{y}}$} & 14 & 0 & 30 & $294 \pm 10 a$ & 0 & 0 \\
\hline & 14 & 6 & 24 & $361 \pm 32 b$ & 0 & 0 \\
\hline & 14 & 12 & 18 & $294 \pm 9 a$ & 0 & 0 \\
\hline & 14 & 18 & 12 & $306 \pm 9 a b$ & 0 & 0 \\
\hline & 14 & 24 & 6 & $305 \pm 6 a b$ & 0 & 0 \\
\hline & 14 & 30 & 0 & $271 \pm 13 \mathrm{a}$ & 0 & 0 \\
\hline
\end{tabular}

Observations were continued for 10 (seeds) and 8 (embryos) weeks after $15-5-15{ }^{\circ} \mathrm{C}$ temperature treatments.

${ }^{\mathrm{z}} \mathrm{n}=10$.

$\mathrm{y}_{\mathrm{n}}=8$.

${ }^{\mathrm{x}}$ Data are expressed as mean \pm SE. Means followed by different letters within a species are significantly different $(P<0.05$, one-way analysis of variance with Tukey's post hoc test).

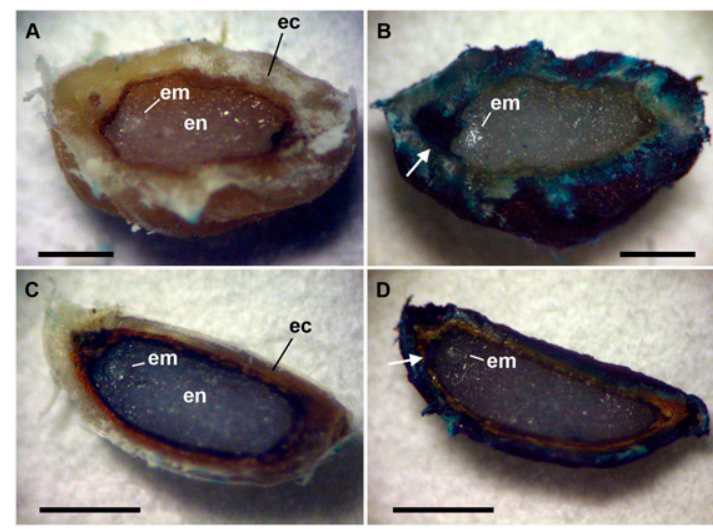

Fig. 2. Water permeability of seeds of Ilex latifolia (A-B) and I. rotunda (C-D). Intact seeds were immersed in distilled water $(\mathbf{A}, \mathbf{C})$ or $1 \%$ methylene blue $(\mathbf{B}, \mathbf{D})$ for $48 \mathrm{~h}$, then longitudinally cut and photographed. The stained testa adjacent to the embryo is indicated by arrows $(\mathbf{B}, \mathbf{D})$. Em = embryo; en $=$ endosperm; ec $=$ endocarp. Scale bars $=1 \mathrm{~mm}$. germination, five types of seeds that differed in the presence of the endocarp, testa, and endosperm (Fig. 1) were cultured on MS medium. The results (Fig. 3A) showed that among these seeds, germinability of isolated embryos was highest; $100 \%$ of embryos germinated within 8 weeks of incubation. Likewise, good germination was observed in quarter-seeds with or without endocarp $(70 \%$ germination within 8 to 10 weeks) followed by half-seeds $(50 \%$ germination within 8 to 10 weeks). No germination was observed when intact seeds were cultured, even 10 weeks after incubation.

When seeds germinated, a radicle and hypocotyl usually appeared on the cut surface of seeds (Fig. 3B). In some quarter-seeds without endocarp, the radicle or hypocotyl penetrated the testa and emerged (Fig. 3C).

Effect of chemical scarification on seed germination. The endocarp of I. latifolia seeds were softened by $\mathrm{NaOH}$ treatment. However, the seeds did not germinate within six months of sowing on wet filter paper and incubating at $25^{\circ} \mathrm{C}$.

Effect of gibberellic acid on seed germination. Seeds of I. latifolia and I. rotunda treated with $\mathrm{GA}_{3}$ did not germinate within six months of sowing on wet filter paper and incubating at $15^{\circ} \mathrm{C}$. No change in embryo length was observed after incubation.

Effect of cold stratification on seed germination. After cold stratification, no change in embryo length was observed in I. latifolia (Table 3). Neither cold-stratified nor non-stratified seeds germinated. When embryos were cultured in vitro, $50 \%$ of nonstratified embryos germinated, whereas the germination frequencies of embryos from seeds stratified for 18 to 30 weeks were $70 \%$ to $80 \%$. In $I$. rotunda, although the lengths of embryos from seeds stratified for 6 weeks increased to $361 \mu \mathrm{m}$, no change in embryo length was observed in other seeds stratified for 12 to 30 weeks. No seeds or embryos germinated.

\section{Discussion}

In the present study, intact seeds of $I$. latifolia and $I$. rotunda did not germinate in any of the experiments. When embryos were cultured in vitro, germination was observed in I. latifolia but not in I. rotunda. Therefore, germinability obviously differed between these species. Embryos of I. latifolia elongated until October and showed good germination in October or later, whereas those of I. rotunda elongated until around November. Although fruits of both species reached maturity in the fall, embryos were apparently in the heart or late-heart stage even in February. Because immaturity of embryos was also reported in several other Ilex species (Chien et al., 2011; Hu et al., 1979; Tsang and Corlett, 2005), immaturity could be a common phenomenon in Ilex.

In I. latifolia, germination frequency decreased transiently in embryos isolated from seeds collected in Sept. 2006 and Oct. 2007. Although it is unclear why transient decrease 
A
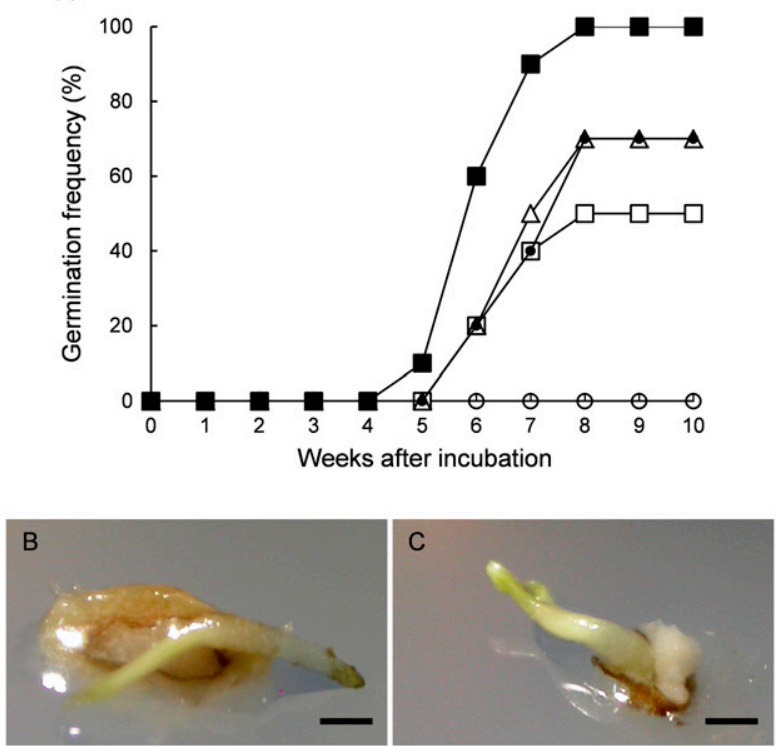

Fig. 3. Germination of Ilex latifolia seeds that differed in the presence of the endocarp, testa, and endosperm. (A) Germination frequencies. Open circles = intact seeds; open squares = half-seeds; open triangles $=$ quarter-seeds; filled circles $=$ quarter-seeds without endocarp; filled squares $=$ embryos . (B-C) Seedling emergence from cut seeds. The radicle and hypocotyl appeared on the cut surface of half-seeds (B), whereas they appeared after penetrating the testa in quarter-seeds without endocarp (C). Scale bars $=1 \mathrm{~mm}$.

of germination frequency was observed, these results implied that during development, embryos increased germinability but underwent transient dormancy.

In I. paraguariensis and I. dumosa, because cut seeds germinated while intact seeds did not or showed delayed germination, the endocarp was suggested to act as a mechanical barrier interfering with tissue expansion (Dolce et al., 2010, 2011). However, subsequent experiments have incompletely addressed the hypothesis. In the present study, a water permeability test using methylene blue suggested that seeds of I. latifolia and I. rotunda could imbibe water. In experiments using five types of I. latifolia seeds that differed in the presence of the endocarp, testa, and endosperm, the radicle or hypocotyl penetrated the testa and emerged only when quarter-seeds without endocarp were cultured in vitro. These results strongly suggested that the endocarp acted as a mechanical barrier to radicle and hypocotyl emergence rather than avoiding water absorption.

Chemical scarification using $\mathrm{NaOH}$ did not allow seed germination in I. latifolia even six months after sowing on wet filter paper. Although further investigation of $\mathrm{NaOH}$ concentrations and treatment duration may be required, Dolce et al. (2010) also reported that scarification using $\mathrm{H}_{2} \mathrm{SO}_{4}$ had no effect on seed germination in I. paraguariensis.

In Arabidopsis, the plant hormone abscisic acid (ABA) synthesized in the endosperm is thought to induce seed dormancy (Lee et al., 2010; Lefebvre et al., 2006). Because germination frequency increased as the sizes of the remaining endosperm and endocarp decreased, we considered that certain inhibitors, such as ABA, contained in the endosperm, testa, and/or endocarp might interfere with seed germination in I. latifolia. This hypothesis is consistent with the suggestion that I. aquifolium, I. cornuta, and I. opaca possess inhibitors in the endosperm and/or membranelike testa attached to the endosperm that are responsible for maintaining embryo dormancy (Hu et al., 1979) but inconsistent with the data for I. paraguariensis and I. dumosa, in which the endosperm did not inhibit embryo development or germination (Dolce et al., 2010, 2011).

Another plant hormone, gibberellin (GA), is known to break seed dormancy and promote germination. GA and ABA are likely to antagonistically interact to control seed dormancy and germination (Chen et al., 2010; Nicolás et al., 1996). Although we treated seeds of I. latifolia and I. rotunda with $\mathrm{GA}_{3}$, they did not germinate. The effects of $\mathrm{GA}_{3}$ on enhancing seed or embryo germination have been tested in several Ilex species. However, $\mathrm{GA}_{3}$ had only slight effects on the germination of embryos with endosperm in I. opaca (Hu et al., 1979) and no effect on germination of intact or bisected seeds in I. paraguariensis (Dolce et al., 2010). In I. maximowicziana, $\mathrm{GA}_{3}$ increased germination rate (shortened germination time) but not germination frequency of intact seeds (Chien et al., 2011). To break seed dormancy, other GAs such as $\mathrm{GA}_{1}, \mathrm{GA}_{4}$, and $\mathrm{GA}_{4}+7$ were reported to be effective in some plant species (Chen et al., 2008, 2010; Chien et al., 1998). In some Ilex species, when a mixture of GAs $\left(\mathrm{GA}_{3}, \mathrm{GA}_{4}\right.$, and $\mathrm{GA}_{7}$ ) was used for embryo culture, germination improved slightly or was unaffected from August to October and inhibited in November and December (Mattis et al., 1995). Nevertheless, GAs other than
$\mathrm{GA}_{3}$ might be effective for breaking seed dormancy in I. latifolia and I. rotunda.

The effects of cold stratification on germination of cut seeds are likely to differ among Ilex species. The germination frequency of cut seeds of I. paraguariensis was increased by cold treatment at $4{ }^{\circ} \mathrm{C}$ for $60 \mathrm{~d}$ (Dolce et al., 2010). Conversely, although germination of cut seeds of $I$. dumosa was not influenced by cold treatment at $4{ }^{\circ} \mathrm{C}$ for $30 \mathrm{~d}$, the frequency was decreased by cold treatment at $4{ }^{\circ} \mathrm{C}$ for $60 \mathrm{~d}$ (Dolce et al., 2011). Cold stratification was not required to break seed dormancy in I. maximowicziana, because cold treatment at $5{ }^{\circ} \mathrm{C}$ for 4 to 8 weeks increased germination rate (shortened germination time) but not germination frequency of intact seeds (Chien et al., 2011). The present study revealed that the germination frequency of $I$. latifolia embryos was increased by cold stratification at $5{ }^{\circ} \mathrm{C}$ for 18 to 30 weeks. Although I. rotunda did not germinate, embryos from seeds cold-stratified at $5^{\circ} \mathrm{C}$ for 6 weeks increased in length, suggesting that embryos collected in February could develop under appropriate cold stratification conditions.

The use of germinating cut seeds, as compared with embryo culture, has been described for I. paraguariensis (Dolce et al., 2010) and $I$. dumosa (Dolce et al., 2011) as follows. Because Ilex embryos are quite small and easily damaged during isolation, the internal seed structure must be precisely known. In addition, embryo culture is very laborious. In vitro culture of cut seeds was easier and more effective than embryo culture. Although cut seeds of I. latifolia germinated less frequently than embryos in this study, we confirmed the convenience of in vitro culture of cut seeds.

In conclusion, seeds of I. latifolia and I. rotunda were difficult to germinate. The results obtained in the present study suggested that factors inhibiting seed germination in I. latifolia were a mechanical barrier by the endocarp and inhibitors contained in the endosperm, testa, and/or endocarp. In I. latifolia, seedlings could be efficiently obtained by culturing embryos and quarterseeds with or without endocarp from seeds collected in November or later, and the germination frequency of embryos could be increased by cold stratification. Germination was not observed in I. rotunda, even when embryos were cold-stratified. Because cold stratification might induce embryo development in I. rotunda, it deserves further investigation in combination with other treatments to obtain seedlings from seeds or embryos.

\section{Literature Cited}

Abe, T. and M. Matsunaga. 2011. Geographic variation in germination traits in Melia azedarach and Rhaphiolepis umbellata. Amer. J. Plant Sci. 2:52-55.

Acebedo, M.M., S. Lavee, J. Liñán, and A. Troncoso. 1997. In vitro germination of embryos for speeding up seedling development in olive breeding programmes. Sci. Hort. 69:207-215. 
Biggs, B.J., M.K. Smith, and K.J. Scott. 1986. The use of embryo culture for the recovery of plants from cassava (Manihot esculenta Crantz) seeds. Plant Cell Tiss. Org. Cult. 6:229-234.

Chee, P.P. 1994. In vitro culture of zygotic embryos of Taxus species. HortScience 29:695697.

Chen, S.Y., C.T. Chien, J.M. Baskin, and C.C. Baskin. 2010. Storage behavior and changes in concentrations of abscisic acid and gibberellins during dormancy break and germination in seeds of Phellodendron amurense var. wilsonii (Rutaceae). Tree Physiol. 30:275-284.

Chen, S.Y., S.R. Kuo, and C.T. Chien. 2008. Roles of gibberellins and abscisic acid in dormancy and germination of red bayberry (Myrica rubra) seeds. Tree Physiol. 28:1431-1439.

Chien, C.T., S.Y. Chen, T.Y. Chien, J.M. Baskin, and C.C. Baskin. 2011. Nondeep simple morphophysiological dormancy in seeds of Ilex maximowicziana from northern (subtropical) and southern (tropical). Taiwan. Ecol. Res. 26:163-171.

Chien, C.T., L.L. Kuo-Huang, and T.P. Lin. 1998. Changes in ultrastructure and abscisic acid level, and response to applied gibberellins in Taxus mairei seeds treated with warm and cold stratification. Ann. Bot. (Lond.) 81:41-47.

Crisosto, C. and E.G. Sutter. 1985. Role of the endocarp in 'Manzanillo' olive seed germination. J. Amer. Soc. Hort. Sci. 110:50-52.

Dolce, N.R., L.A. Mroginski, and H.Y. Rey. 2010. Endosperm and endocarp effects on the Ilex paraguariensis A. St.-Hil. (Aquifoliaceae) seed germination. Seed Sci. Technol. 38:441-448.

Dolce, N.R., L.A. Mroginski, and H.Y. Rey. 2011. Enhanced seed germination of Ilex dumosa R. (Aquifoliaceae) through in vitro culture of cut pyrenes. HortScience 46:278-281.

Galle, F.C. 1997. Hollies: The genus Ilex. Timber Press, Portland, OR.

Goi, M., A. Hasegawa, and Y. Nishihara. 1978. Studies on seed germination of Ilex rotunda Thunb. I. Flower formation, flowering, fruiting and seed development. Technical Bulletin of Faculty of Agriculture, Kagawa University 30:53-59 [in Japanese with English summary].

$\mathrm{Hu}, \mathrm{C} . \mathrm{Y} .1975$. In vitro culture of rudimentary embryos of eleven Ilex species. J. Amer. Soc. Hort. Sci. 100:221-225.

Hu, C.Y., F. Rogalski, and C. Ward. 1979. Factors maintaining Ilex rudimentary embryos in the quiescent state and the ultrastructural changes during in vitro activation. Bot. Gaz. 140:272279.

Lee, K.P., U. Piskurewicz, V. Turecková, M. Strnad, and L. Lopez-Molina. 2010. A seed coat bedding assay shows that RGL2-dependent release of abscisic acid by the endosperm controls embryo growth in Arabidopsis dormant seeds. Proc. Natl. Acad. Sci. USA 107: 19108-19113.

Lefebvre, V., H. North, A. Frey, B. Sotta, M. Seo, M. Okamoto, E. Nambara, and A. Marion-Poll. 2006. Functional analysis of Arabidopsis NCED6 and $N C E D 9$ genes indicates that ABA synthesized in the endosperm is involved in the induction of seed dormancy. Plant J. 45:309319.

Liang, Y., W. Ma, J. Lu, and Y. Wu. 2001. Comparison of chemical compositions of Ilex latifolia Thumb and Camellia sinensis L. Food Chem. 75:339-343.

Luna, C., P. Sansberro, L. Mroginski, and J. Tarragó. 2003. Micropropagation of Ilex dumosa (Aquifoliaceae) from nodal segments in a tissue culture system. Biocell 27:205-212.

Mattis, P.R., H.J. Swartz, and G. Eisenbeiss. 1995 Development of embryo rescue and shoot regeneration techniques in Ilex. J. Environ. Hort. 13:164-168

Murashige, T. and F. Skoog. 1962. A revised medium for rapid growth and bio assays with tobacco tissue cultures. Physiol. Plant. 15:473497.

Nicolás, C., G. Nicolás, and D. Rodríguez. 1996. Antagonistic effects of abscisic acid and gibberellic acid on the breaking of dormancy of
Fagus sylvatica seeds. Physiol. Plant. 96:244250.

Orozco-Segovia, A., J. Márquez-Guzmán, M.E. Sánchez-Coronado, A. Gamboa de Buen, J.M. Baskin, and C.C. Baskin. 2007. Seed anatomy and water uptake in relation to seed dormancy in Opuntia tomentosa (Cactaceae, Opuntioideae). Ann. Bot. (Lond.) 99:581-592.

Raghavan, V. 2003. One hundred years of zygotic embryo culture investigations. In Vitro Cell. Dev. Biol. 39:437-442.

Rey, H.Y., P.A. Sansberro, M.M. Collavino, J.R. Daviña, A.M. Gonzàlez, and L.A. Mroginski. 2002. Colchicine, trifluralin, and oryzalin promoted development of somatic embryos in Ilex paraguariensis (Aquifoliaceae). Euphytica 123:49-56.

Sansberro, P.A., H.Y. Rey, and L.A. Mroginski. 2001. In vitro culture of zygotic embryos of Ilex species. HortScience 36:351-352.

Sansberro, P.A., H.Y. Rey, L.A. Mroginski, and M.M. Collavino. 1998. In vitro culture of rudimentary embryos of Ilex paraguariensis: Responses to exogenous cytokinins. J. Plant Growth Regul. 17:101-105.

Sharma, D.R., R. Kaur, and K. Kumar. 1996. Embryo rescue in plants-A review. Euphytica 89:325-337.

Sun, Y.C., Y.J. Zhang, K. Wang, and X.J. Qiu. 2006. $\mathrm{NaOH}$ scarification and stratification improve germination of Iris lactea var. chinensis seed. HortScience 41:773-774.

Tezuka, T., H. Yokoyama, H. Tanaka, S. Shiozaki, and M. Oda. 2012. Seed and embryo germination in Ardisia crenata. J. Bot. 2012:679765.

Tsang, A.C.W. and R.T. Corlett. 2005. Reproductive biology of the Ilex species (Aquifoliaceae) in Hong Kong, China. Can. J. Bot. 83:16451654.

Tsuyuzaki, S. and C. Miyoshi. 2009. Effects of smoke, heat, darkness and cold stratification on seed germination of 40 species in a cool temperate zone in northern Japan. Plant Biol. 11:369-378. 